\title{
Do Forward- and Backward-Traveling Waves Occur Within the Cochlea? Countering the Critique of Nobili et al.
}

\author{
Christopher A. Shera, ${ }^{1,2}$ Arnold Tubis, ${ }^{3,4}$ and Carrick L. Talmadge ${ }^{5}$ \\ ${ }^{1}$ Eaton-Peabody Laboratory of Auditory Physiology, Massachusetts Eye and Ear Infirmary, Boston, MA 02114, USA \\ ${ }^{2}$ Department of Otology and Laryngology, Harvard Medical School, Boston, MA 02115, USA \\ ${ }^{3}$ Department of Physics, Purdue University, West Lafayette, IN 47907, USA \\ ${ }^{4}$ Institute for Nonlinear Science, University of California, San Diego, La Jolla, CA 92093, USA \\ ${ }^{5}$ National Center for Physical Acoustics, University of Mississippi, University, MI 38677, USA
}

Received: 16 September 2003; Accepted: 26 April 2004; Online publication: 6 August 2004

\section{ABSTRACT}

The question of whether or not forward- and backward-traveling waves occur within the cochlea constitutes a long-standing controversy in cochlear mechanics recently brought to the fore by the problem of understanding otoacoustic emissions. Nobili and colleagues articulate the opposition to the traveling-wave viewpoint by arguing that wave-equation formulations of cochlear mechanics fundamentally misrepresent the hydrodynamics of the cochlea [e.g., Nobili et al. (2003) J. Assoc. Res. Otolaryngol. 4:478494]. To correct the perceived deficiencies of the wave-equation formulation, Nobili et al. advocate an apparently altogether different approach to cochlear modeling-the so-called "hydrodynamic" or "Green's function" approach-in which cochlear responses are represented not as forward- and backward-traveling waves but as weighted sums of the motions of individual basilar membrane oscillators, each interacting with the others via forces communicated instantaneously through the cochlear fluids. In this article, we examine Nobili and colleagues' arguments and conclusions while attempting to clarify the broader issues at stake. We demonstrate that the one-dimensional wave-equation formulation of cochlear hydrodynamics does not misrepresent long-

Correspondence to: Christopher A. Shera $\cdot$ Eaton-Peabody Laboratory - Massachusetts Eye and Ear Infirmary • 243 Charles Street • Boston, MA 02114. Telephone: (617) 573-4235; Fax: (617) 720-4408; email: shera@epl.meei.harvard.edu range fluid coupling in the cochlea, as claimed. Indeed, we show that the long-range component of Nobili et al.'s three-dimensional force propagator is identical to the hydrodynamic Green's function representing a one-dimensional tapered transmission line. Furthermore, simulations that Nobili et al. use to discredit wave-equation formulations of cochlear mechanics (i.e., cochlear responses to excitation at a point along the basilar membrane) are readily reproduced and interpreted using a simple superposition of forward- and backward-traveling waves. Nobili and coworkers' critique of wave-equation formulations of cochlear mechanics thus appears to be without compelling foundation. Although the traveling-wave and hydrodynamic formulations impose strikingly disparate conceptual and computational frameworks, the two approaches ultimately describe the same underlying physics.

Keywords: inner ear, middle ear, otoacoustic emissions, cochlear mechanics

\section{INTRODUCTION}

Since before von Békésy first visualized the wavelike motion of the cochlear partition, the mechanical responses of the cochlea have been understood using concepts borrowed from the description of wave propagation in electrical transmission lines (e.g., 
Wegel and Lane 1924; Zwislocki-Mościcki 1948; Peterson and Bogert 1950; de Boer 1980; Lighthill 1981). In recent years, however, Nobili and colleagues have criticized wave-equation formulations of cochlear mechanics on the grounds that they fundamentally misrepresent the hydrodynamics of the cochlea (Nobili 2000; Nobili et al. 1998, 2003a,b). They write, for example, that "the solutions of the BM [basilarmembrane] motion equation are not of the bi-directional wave-propagation type" and can "in no way ... be represented as the superposition of progressive and regressive wave components."

To remedy the putative deficiencies of the waveequation formulation, Nobili et al. advocate the socalled "hydrodynamic" approach to cochlear modeling. In this approach, exemplified by Nobili et al.'s "BM integrodifferential equation" (Mammano and Nobili 1993), cochlear responses are represented not as forward- and backward-traveling waves but as the summed activity of the individual BM oscillators, each of which interacts with every other via hydrodynamic forces communicated nearly instantaneously through the cochlear fluids. Nobili explains that in contrast to a transmission line and its manifest support for forward- and backward-traveling waves, "the hydrodynamic model of the cochlea is based on an integral differential equation... [that captures] the hydrodynamic coupling between oscillating elements. The solutions to this equation are not similar to the types that describe sound- or light-wave propagation" (quoted in Allen 2003, p. 586).

As a corollary of their critique, Nobili et al. (2000, 2003a, 2003b) condemn existing models of otoacoustic emissions (OAEs), especially those that incorporate the language of wave propagation and reflection (e.g., Shera and Zweig 1993; Zweig and Shera) 1995; Talmadge et al. 1998, 2000). They write, for example, that although wave-reflection models of OAEs may seem "conceptually appealing" on the surface, their underlying tenets are, "in reality, ... difficult to reconcile ... with the physics of the cochlea." Indeed, Nobili et al. contend that "no wave reflection takes place within the cochlea" so that "talking about wave 'reflection' ... is physically inappropriate and even conceptually misleading."

Nobili et al.'s critique clearly cuts a wide swath through cochlear mechanics, and their analysis nourishes a perennial controversy (e.g., Allen 2003, p. 583ff). Assessing the validity of Nobili et al.'s arguments therefore has broad implications, not only for interpreting otoacoustic emissions, where the issues appear especially salient, but also for understanding the most basic operation of the cochlea. In this article we therefore address the question: Does Nobili et al.'s analysis of cochlear hydrodynamics invalidate waveequation formulations of cochlear mechanics? In a nutshell, we find that Nobili et al.'s characterization of the wave-equation formulation is incorrect.

We emphasize, however, that our findings do not negate Nobili et al.'s critique by demonstrating that the hydrodynamic approach is itself invalid. Rather, we resolve and synthesize the two approaches, arguing that the wave-equation and hydrodynamic formulations of cochlear mechanics are but different mathematical representations of the same underlying physics-a full understanding necessarily subsumes both. What appears invalid, then, is neither the traveling-wave nor the hydrodynamic view of cochlear mechanics, but Nobili et al.'s false dichotomy that distinguishes the two on physical grounds. Although both formulations provide valid representations of the physics of the cochlea, the two approaches are attended by strikingly different conceptual and computational frameworks. Since any choice between the two depends on the nature of the questions to be addressed, we argue that the wave-equation formulation provides compelling advantages, at least in the context of modeling OAEs.

\section{ARE WAVE-EQUATION FORMULATIONS UNPHYSICAL?}

\section{Nature of the fluid coupling}

Nobili et al. (2003a) launch their critique of waveequation formulations of cochlear mechanics by arguing that transmission-line models fundamentally misrepresent the hydrodynamics of the cochlea. In particular, they claim that transmission-line models "reduce fluid coupling to a sort of local interaction, thus failing to represent adequately its long-range character." This same criticism also surfaces in a recent review (Nobili et al. 1998), in which the authors remark that "instantaneous hydrodynamic coupling among different basilar membrane portions has a long-range character that is only approximately represented by nearest-neighbour transmission-line interactions." As we explain below, these statements misrepresent the nature of the fluid coupling in the one-dimensional model.

Throughout their work, Nobili et al. characterize the fluid coupling between different cochlear locations with the "hydrodynamic Green's function," $G(x, \bar{x})$, whose value represents the strength of the fluid-mediated interaction between locations $x$ and $x$ along the BM. The function $G(x, \bar{x})$ in their model generally consists of a small "spike," centered at $x=\bar{x}$, superimposed on a prominent "hump" that extends throughout most of the cochlea (e.g., Nobili et al. 2003a, Fig. 2d; see also Mammano and Nobili 1993, Fig. A3). This hump characterizes the nearinstantaneous long-range fluid coupling-a conse- 
quence of the near-incompressibility of the cochlear fluids-between points widely separated along the partition. Since Nobili et al. describe fluid coupling in the long-wave model as "a sort of local interaction," their readers might reasonably expect the corresponding one-dimensional Green's function to lack the important long-range component, to be, in effect, "all spike and no hump." The truth, however, is just the opposite: As we demonstrate in the Appendix, the one-dimensional hydrodynamic Green's function differs from its higher-dimensional counterparts not in its characterization of long-range fluid forces, but in its description of the local, shortrange coupling. Nobili et al. implicitly acknowledge this fact when they concede that a "rough equivalence between transmission line and hydrodynamic models of the cochlea can be established ... for the simplified geometry of the box model."

\section{Validity in realistic geometries}

As the previous quotation suggests, Nobili et al.'s basic objection to the transmission-line model ultimately seems to arise from its putative restriction to the idealized geometry of the box model. Indeed, Nobili et al.'s model was first developed precisely to overcome perceived hydrodynamic "deficiencies affecting previous 'box' models of the cochlea" (Mammano and Nobili 1993). In a nutshell, their argument against the transmission line appears to be that no matter how well the model may represent long-range fluid coupling in an imagined rectangular box, the transmission-line model must necessarily fail to capture the hydrodynamics operating in the more complex, tapered geometry of the real cochlea. Thus, Nobili et al. argue that although the concepts of wave propagation and reflection may apply in idealized hypothetical situations, transmission-line models cannot describe anything resembling the physics of an actual ear (e.g., Nobili et al. 2003a,b).

To emphasize the impossibility of converting their "BM integrodifferential equation" into a partial differential equation representing a transmission line, Mammano and Nobili (1993) assert that in models with realistic cochlear geometry "the long-range action of the Green's function ... cannot be canceled by multiple differentiation and the full integral-equation technique must be applied." In other words, Nobili et al. argue that no approximate equivalence with a transmission line exists for models with realistic geometry. But as we demonstrate in the Appendix, this argument is incorrect. In the Appendix we use the one-dimensional transmission-line model to derive the Green's function for a tapered cochlea with asymmetric scalae areas. The resulting Green's function is identical to the long-range component of the
Green's function (or "force propagator") obtained by Mammano and Nobili (1993) for their threedimensional model. Nobili et al.'s model therefore shares with the much maligned box model a key attribute: With respect to long-range fluid coupling, both models are equivalent to one-dimensional transmission lines.

We reemphasize that the approximation involved in reducing two- and three-dimensional models to one-dimensional transmission lines has nothing to do with long-range fluid coupling, which is always effectively one dimensional, even in realistic tapered geometries. Instead, the approximation involves the nature of the short-range coupling represented by the spike in the Green's function at $x=\bar{x}$ (Allen 1977). The nature of this spike is determined by the effective dimensionality of the fluid motion in the vicinity of the cochlear partition. The spike is absent in one dimension and appears as a logarithmic singularity in two dimensions (Allen 1977). In three dimensions, the Green's function $G(x, \bar{x})$ that Nobili et al. employ does not really exist as such. When the pressure varies in the radial (or $y$ ) direction across the partition, the Green's function has the form $G(x, y ; \bar{x}, \bar{y})$. An "effective" Green's function with the reduced form $G(x, \bar{x})$ can, however, be defined by appropriate averaging along the radial direction. Although the nature of the resulting singularity may depend on precisely how this averaging is accomplished, we generally expect a singularity similar to that of the two-dimensional case. None of these local interactions appreciably affect the long-range fluid coupling.

\section{Interpretation of forces applied to the basilar} membrane

Finally, Nobili et al. (2003a; see also Allen 2003, p. 586) round out their case against wave reflection with a comparative analysis of the "BM integrodifferential motion equation ... and the hyperbolic [wave] equation that governs sound, light, and surface wave propagation.' In Figure 6 of Nobili et al. (2003a), they show their cochlear model's response to a sinusoidal force applied directly to the BM. Aside from a few prominent amplitude notches and corresponding distortions in the phase, the resulting response generally resembles that obtained when the stimulus is applied at the stapes. "In particular," they write, "both [response] profiles affect, with appreciable amplitude, the same limited region of the cochlear partition"; in other words, the effects of the applied force "remain confined to the neighborhood of the CF site."

Nobili et al. contrast this behavior with the longrange propagation effects found in systems governed 


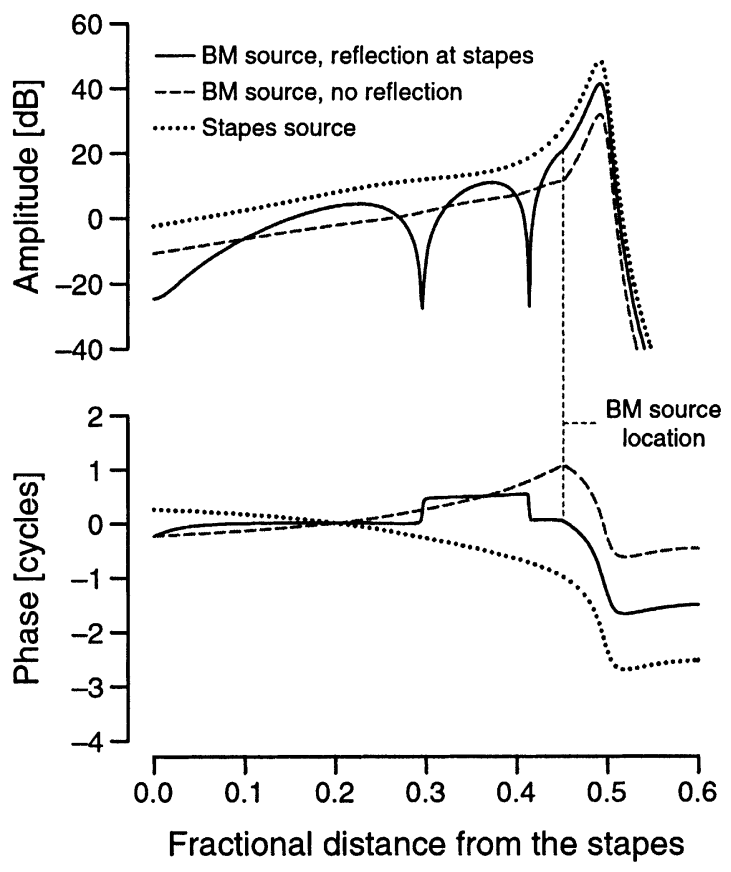

FIG. 1. Response elicited by a stimulus applied to the basilar membrane in a transmission line model of the cochlea. As in Figure 6 of Nobili et al. (2003a), the solid lines show the amplitude and phase of the response to a sinusoidal stimulus $(\sim 2 \mathrm{kHz})$ applied at the BM location $\bar{x}$ indicated by the labeled vertical line spanning the top and bottom panels. In this case, the impedance mismatch at the cochlear boundary with the middle ear results in a stapes reflection coefficient with the value $R_{\text {stapes }}=-0.8$. Although the precise depths and locations of the amplitude notches and phase distortions depend on the value of $R_{\text {stapes, }}$ other nonzero values give qualitatively similar results. For comparison, the dashed lines show the response to the same stimulus when the middle-ear impedance is changed to simulate a perfectly reflectionless boundary $\left(R_{\text {stapes }}=0\right)$. The dotted lines show the forward-traveling wave elicited when the stimulus is applied at the stapes. As in Nobili et al. (2003a), the amplitudes represented by the solid and dotted lines were normalized to similar peak values and then offset slightly for clarity. BM velocity responses were computed using the model of Zweig (1991), with parameter values modified to roughly approximate the responses seen in Nobili et al.'s simulations.

by wave equations, such as the voltage in an electrical transmission line, ripples on the surface of a pond, or the displacements of a vibrating guitar string. In these cases, a local force (e.g., a pluck to the guitar string) generates both forward- and backward-traveling waves that "proceed towards the ends of the integration domain, where reflection can occur.' Thus, Nobili et al. argue that the localized cochlear response predicted by their "BM integrodifferential equation" differs profoundly from the long-range propagation effects characteristic of systems described by wave equations. Indeed, "the solutions of the BM motion equation are not of the bi-directional wave-propagation type" and can "in no way ... be represented as the superposition of progressive and regressive wave components." Since the mechanical perturbations needed to produce OAEs can be modeled using an appropriate distribution of forces acting on the BM, Nobili et al. conclude from their analysis that "internal TW [traveling-wave] reflections could hardly be invoked to explain the generation of OAEs."

Although intended to refute the wave-equation formulation and thereby "clarify the rationale underlying [their hydrodynamic] ... approach," Nobili et al.'s simulations actually provide a compelling illustration of the wave formulation's explanatory power. Nobili et al. rest their case on the implication that their analysis of forces applied to the BM contradicts all wave-equation formulations of cochlear mechanics ("our model disclosed a different [i.e., non-wave-like] behavior"). But our Figure 1 demonstrates that their findings are easily reproduced using a simple transmission-line model. Furthermore, the analytic tractability of the transmission-line model provides a straightforward interpretation of the results in terms of wave propagation and reflection, an interpretation almost entirely obscured by Nobili et al.'s computational Green's function approach.

To understand Nobili et al.'s results, consider approximating the cochlea as a one-dimensional hydromechanical transmission line (e.g., ZwislockiMościcki 1948; Peterson and Bogert 1950; de Boer 1980, 1984; Zweig 1991). An oscillating point source introduced at location $\bar{x}$ generates waves that propagate away from the source in both directions. If the source frequency is less than the local CF, the forward-traveling wave propagates apically toward its characteristic place, beyond which it is strongly attenuated. (For simplicity, we assume that the mechanics vary smoothly with position so that no wave scattering occurs about the peak of the response.) The wave leaving the source in the backward direction travels toward the base of the cochlea. When it reaches the stapes, the wave is partially reflected by the impedance mismatch with the middle ear. This reflection generates a new forward-traveling wave that propagates back toward $\bar{x}$, whereupon it combines with the forward-traveling wave emanating directly from the source.

Whereas the cochlear region apical to the source at $\bar{x}$ contains only a single, forward-traveling wave, the basal region between the stapes and $\bar{x}$ generally contains a wave traveling in each direction. The complex amplitude of the pressure difference across the partition therefore has the form

$$
P(x)= \begin{cases}a_{+} W_{+}(x) & \text { for } x>\bar{x} \\ b_{-} W_{-}(x)+b_{+} W_{+}(x) & \text { for } x\end{cases}
$$

where the functions $W_{ \pm}(x)$ represent the forward-and backward-traveling-wave solutions to the transmission- 
line equations [i.e., the pressure "basis waves" (Shera and Zweig 1991a; Talmadge et al. 1998)] and the three complex constants $a_{+}$and $b_{ \pm}$are determined by requiring that $P(x)$ satisfy both the basal boundary condition at the stapes and continuity of pressure and conservation of volume velocity at $\bar{x}$. For example, the basal boundary condition implies that $b_{+}=b_{-} R_{\text {stapes }}$, where $R_{\text {stapes }}$ is the stapes reflection coefficient for backward-traveling waves [the basis waves are normalized so that $\left.W_{ \pm}(0)=1\right]$. The $\mathrm{BM}$ velocity is everywhere proportional to the product of the driving pressure $P(x)$ and the local admittance of the cochlear partition.

In the cochlear region apical to the source $(x>\bar{x})$, the response to the applied stimulus is a simple forward-traveling wave, $a_{+} W_{+}(x)$. The envelope of this wave varies with position in the familiar way, increasing up to the wave's characteristic place and declining steeply beyond. The peak in the response envelope results from the combined effects of cochlear amplification and damping, as well as the spatial variation in the impedance of the partition. Thus, in contradiction to Nobili et al.'s analysis, the observation that the response peaks in a region straddling the CF site-just as it does when the stimulus is delivered at the stapes-provides no evidence against the underlying wave equation; it simply reflects the well-established fact that traveling-wave amplitudes vary strongly with position.

In the region basal to the source $(x<\bar{x})$, the response is more complicated, with its qualitative form dependent on the middle-ear boundary condition. In the simplest case, the backward-traveling wave $b_{-} W_{-}(x)$ incident upon the stapes remains unreflected $\left(R_{\text {stapes }}=0\right)$. As a result, $b_{+}=0$ and the response consists of a wave propagating away from the source at $\bar{x}$ toward the stapes (Fig. 1 dashed line). When $R_{\text {stapes }}$ is nonzero, however, the backward-traveling wave is reflected back into the cochlea, and the pressure between the stapes and source at $x$ acquires a standing-wave component (Fig. 1, solid line). The two wave components, $b_{+} W_{+}(x)$ and $b_{-} W_{-}(x)$, then beat against each other as their respective phases rotate along the cochlea. This interference creates the observed amplitude notches and corresponding phase distortions, which are spaced at intervals determined by the spatial variation of the wavelength.

In summary, the relevant features of the responses shown in Figure 1-a normal-looking traveling wave in the region apical to the stimulus location at $\bar{x}$ and the amplitude notches and phase distortions basal to $\bar{x}$-are all readily understood in terms of forward-and backward-traveling waves. Although Nobili et al. provide no account of the origin of these features, our analysis demonstrates that they result from the same wave propagation, reflection, and interference effects whose existence Nobili et al. sought to deny.

\section{DISCUSSION}

The striking similarities between Nobili et al.'s computations and the wave pattern predicted by a simple transmission-line model are no coincidence (compare Fig. 6 of Nobili et al. 2003a with our Fig. 1). Our results establish that the long-range fluid coupling underlying Nobili et al.'s integrodifferential equation is identical to that in a one-dimensional, tapered transmission line (see the Appendix). The waveequation and Green's function formulations are not, as Nobili et al. imply, two fundamentally different models based on disparate descriptions of cochlear hydrodynamics but two different mathematical representations of a single model based on Newton's laws. Since both formulations represent the same physics, both must ultimately yield the same solutions.

Although the wave-equation and Green's function formulations provide physically equivalent descriptions of long-range fluid coupling in the cochlea, the conceptual and computational frameworks that accompany them are remarkably disparate. Indeed, the two viewpoints are so dissimilar that Nobili et al. see no contradiction in embracing one while denying the validity of the other. Nobili et al. (2003a) condemn the transmission-line view (in which "delays between input and output in the ear canal are interpreted as travel time of back-propagating waves") by arguing that these delays are actually "due to the interplay of BM elasticity and the kinetic energy of the hydrodynamic field." But Nobili et al. perceive sharp distinctions where none exist; their hydrodynamic explanation merely reiterates the physics of the transmission line without using the word "wave." Nobili et al.'s arguments reveal less about cochlear mechanics than they do about the seductive power of alternative conceptual frameworks that allow an underlying physical equivalence to go unrecognized.

Although both wave-equation and hydrodynamic approaches might profitably be explored, any rational choice between the two hinges on the questions to be addressed. As discussed above, Nobili et al.'s work in cochlear modeling began with an elegant treatment of cochlear hydrodynamics that embraced the full three-dimensional tapered geometry of the cochlear scalae (Mammano and Nobili 1993). For this original application, the computational Green's function approach may have certain advantages. In the present context, however, Nobili et al.'s conviction that the transmission-line model distorts the physics of the cochlea fosters a methodology evidently far from optimal for investigating OAEs: "[O] ur model," they write, "uses a realistic representation of human cochlear geometry, leaving us no option but to use the full integrodifferential form of the BM motion equation." 
By carrying over a computational framework originally adopted to address an altogether different problem, Nobili et al. unnecessarily limit their conceptual horizons. When one represents the solution to the cochlear mechanics equations as the instantaneous sum of a large number of oscillating point sources, it is neither easy to visualize nor even natural to talk about the organized, collective patterns (e.g., the forward- and backward-traveling waves) that emerge when all the individual oscillations have been appropriately combined and allowed to interfere with one another. Although not prohibitive in principle, the Green's function approach inevitably diverts precious intellectual and computational resources to the task of calculating a vast array of oscillatory responses, most of which ultimately cancel one another out. In this way, the Green's function representation appears fundamentally mismatched to the problem at hand; as such it may do more to hinder the understanding than to advance it, at least in the context of modeling OAEs. The simple picture of oscillating point sources that attends the Green's function approach doubtless serves the intuition well when the number of participating sources remains small; but visualizing the collective response of the extended, irregular array of asynchronous oscillators responsible for OAEs quickly transcends the intuitive grasp.

By contrast, the basic conceptual and computational atoms of the wave-equation formulation are not the myriad individual oscillators, each interacting with the others instantaneously through the fluids, but the traveling waves that emerge as their collective response (e.g., Békésy 1960; Ren 2002). As a result, the wave-equation framework for modeling OAEs has the invaluable heuristic advantage that the otoacoustic phenomena of primary interest-all of which involve energy going in, interacting in some way, and then coming out again-are immediately and transparently represented in the mathematics. Nobili et al. assert that "talking about wave 'reflection' ... in the cochlea is physically inappropriate and even conceptually misleading," but by adopting the wave-equation framework we merely embrace the basic principle of epistemology so vividly elucidated by the American labor leader Walter Reuther: "If it looks like a duck, walks like a duck, and quacks like a duck, then it just may be a duck.'

Although no less physically appropriate than the Green's function alternative, the wave-equation formulation often provides considerably more aid to the intuition. Contrast, for example, the representations of the middle-ear boundary condition in the two approaches. In the Green's function formulation, this boundary condition is incorporated into the equations in a manner that not only requires extensive numerical computation to implement but also masks its functional significance [see Nobili et al. (2003a) and the Appendix]. In the wave-equation approach, however, the boundary condition is incorporated by means of the reflection coefficient $R_{\text {stapes }}$ as described above. The conceptual clarity of this formulation greatly facilitates the interpretation of model responses (e.g., Nobili et al.'s simulations of the BM response to point excitation along the partition). Furthermore, the formulation encourages systematic manipulation of the stapes boundary condition for the purposes of investigating its effects on the solution (e.g., the case $R_{\text {stapes }}=0$ shown in Fig. 1). Again, nothing about the Green's function approach is physically incorrect, but regarding it as the one true path to cochlear mechanics necessarily means that insights afforded by the complementary view go unappreciated.

Nobili et al.'s discussion of spontaneous otoacoustic emissions (SOAEs) could have benefited from just this sort of alternative perspective. By failing to recognize that the phase distortions evident in Figure 6 of their 2003a paper reflect the presence of intracochlear standing waves (see our Fig. 1), Nobili et al. mischaracterize the origin of the SOAEs produced by their model (see their Appendix). Overlooking the fundamental role played by the middle-ear boundary condition in the generation of these sounds (e.g., Kemp 1979; Talmadge and Tubis 1993; Shera 2003), Nobili et al. attribute SOAEs not to global standingwave resonances but to a local "unbalancing" of undamping caused by lateral rebound forces transmitted via fluid coupling from adjoining oscillators. Had Nobili et al. been able to modify their middle-ear boundary condition in a convenient and functionally interpretable way-indeed, had their conceptual viewpoint not implicitly discouraged any consideration of collective, wave-interference effects-their account of spontaneous emissions might have been considerably enriched.

Although the equivalence of long-range fluid coupling in cochleae of realistic geometry and in simple, tapered transmission lines may have gone previously unrecognized, the conclusion that transmission-line models do not distort the physics of longrange fluid coupling should come as no surprise. Indeed, the result can be guessed immediately by considering the cochlear mechanical equivalent of a spherical cow, namely, a symmetric box model in which the BM impedance is both stiffness-dominated and in dependent of position, so that transpartition pressure waves travel without dispersion. If the box is sufficiently squat (or the BM sufficiently stiff), the constant wavelength of the pressure-difference wave will be long compared to the heights of the scalae. In other words, the long-wavelength approximation applies, and the hydrodynamics of the system are 
captured almost exactly by the one-dimensional transmission-line equations. When we imagine slowly relaxing these simplifying assumptions to more faithfully represent the mechanics of the cochlea, we find that the long-wavelength approximation begins to break down, but only in the region about the peak of the traveling wave. Although equations that were exact in the squat box model become approximate in more realistic geometries, no violations of the laws of physics are surreptitiously introduced and the character of long-range fluid coupling remains unchanged.

What about the short-range fluid coupling? As discussed above, one-dimensional models clearly simplify cochlear hydrodynamics in the "short-wave" region about the peak of the traveling-wave envelope. Readers may wonder about the implications of this simplification for the generation of reflection-source OAEs as described by the theory of coherent reflection filtering (Shera and Zweig 1993; Zweig and Shera 1995; Talmadge et al. 1998). For ease of analysis, the theory was first developed by applying the framework of the one-dimensional, long-wave model in the shortwave region near the traveling-wave peak, where most of the wave reflection due to coherent scattering from cochlear inhomogeneities occurs. This apparent inconsistency can be remedied by generalizing the perturbative theory of coherent scattering to the case of two-and three-dimensional cochlear models, while still retaining the physical picture of traveling waves (Talmadge et al. 2001; Shera et al. 2004). Although a single differential equation for the transpartition pressure cannot generally be obtained in higherdimensional models, approximate (e.g., WKB type) solutions can nevertheless be derived with travelingwave attributes similar to those in the one-dimensional case. Reassuringly, the basic predictions of the one-dimensional theory survive intact. The fundamental reason for this is simple: The theory indicates that although the mechanisms of coherent scattering depend strongly on functional characteristics of the traveling-wave peak such as its height, width, and wavelength, they remain relatively insensitive to details of the biophysical and hydromechanical processes that determine how that peak originates (Zweig and Shera 1995). To paraphrase Zweig (1991), when modeling OAEs it appears more important, in the hierarchy of approximations, to accurately approximate the form of the traveling wave than to work with the correct number of spatial dimensions.

In the introduction to his book on the mathematics of wave motion, G.B. Whitham (1974) wrote

There appears to be no single precise definition of what exactly constitutes a wave. Various restrictive definitions can be given, but to cover the whole range of wave phenomena it seems preferable to be guided by the intuitive view that a wave is any recognizable signal that is transferred from one part of the medium to another with a recognizable velocity of propagation.

Nobili et al. may disagree, preferring to reserve the language of wave propagation and reflection for some hypothetical cochlea in which the transmissionline equations provide an exact description of the mechanics rather than simply an approximate one. But all useful models are necessarily approximate, and an artificial semantic restriction does nothing to negate the demonstrated, quantitative success of the concepts of wave propagation and reflection for understanding cochlear mechanics.

\section{SUMMARY}

We have shown that transmission-line models successfully capture long-range fluid coupling in the cochlea, contrary to the claims of Nobili et al. (1998, 2000, 2003a, 2003b). Indeed, we have established that the long-range component of Nobili et al.'s threedimensional force propagator is functionally equivalent to the hydrodynamic Green's function of a onedimensional tapered transmission line (see the Appendix). Furthermore, we have demonstrated that simulations Nobili et al. use to discredit wave-propagation models (i.e., BM responses to point excitation along the cochlear partition) are most readily obtained and interpreted using the ideas of wave propagation and reflection in a simple transmission line. Nobili et al.'s critique of wave-equation formulations of cochlear mechanics thus appears to be without compelling foundation. Although both "hydrodynamic" and wave-equation formulations can provide valid representations of the physics of the cochlea, the two formulations impose strikingly different conceptual and computational frameworks. We argue that the wave-equation formulation provides compelling advantages in the context of modeling OAEs.

\section{ACKNOWLEDGMENTS}

We thank Jont Allen, Egbert de Boer, Paul F. Fahey, John J. Guinan, Jr., Radha Kalluri, and Robert H. Withnell for valuable discussions and suggestions. Five reviewers, including Renato Nobili and Fabio Mammano, provided helpful comments on the manuscript. Finally, we thank an anonymous reviewer for bringing the quote by Whitham to our attention. This work was supported by grants R01 
DC03687 and R29 DC03094 from the NIDCD, National Institutes of Health.

\section{APPENDIX A: FLUID COUPLING IN THE TRANSMISSION-LINE MODEL}

In this Appendix we use the long-wavelength approximation to derive the equations describing a tapered cochlea with asymmetric scalae areas. We briefly sketch out how these equations can equivalently be represented using either a wave-equation or a hydrodynamic Green's function formulation. Although both approaches capture the same underlying physics, the mathematical representations they employ are very different. Finally, we establish that the long-range component of Nobili et al.'s threedimensional force propagator is functionally equivalent to the Green's function of a one-dimensional, tapered transmission line.

We begin by generalizing Eq. (1) of Talmadge et al. (1998) describing the symmetric box model to the case where the scalae areas vary with position (e.g., Shera and Zweig 1991b). The pressure difference $p(x, t)$ across the cochlear partition (scala vestibuli minus scala tympani) satisfies the equation

$$
\frac{1}{S} \frac{\partial}{\partial x}\left[S \frac{\partial p}{\partial x}\right]=\rho_{0} b \dot{v}_{\mathrm{bm}} / S
$$

, where $x$ is the distance from the stapes; $t$ is the time and the dot represents a time derivative; $v_{\mathrm{bm}}(x, t)$ is the $\mathrm{BM}$ velocity (positive into the scala tympani); $\rho_{0}$ is the cochlear fluid density; $b(x)$ is the effective $\mathrm{BM}$ width; and $S(x)$ is the effective scala cross-sectional area (Zwislocki 1965; Dallos 1973), given by

$$
S=\frac{S_{\mathrm{v}} S_{\mathrm{t}}}{S_{\mathrm{v}}+S_{\mathrm{t}}}
$$

, where $S_{\mathrm{v}}(x)$ and $S_{\mathrm{t}}(x)$ are the areas of the scala vestibuli and tympani, respectively.

Equation (A1) follows from Newton's laws applied to fluid flow in tapered tubes (e.g., Peterson and Bogert 1950; Shera and Zweig 1991b). In the longwavelength approximation, the pressures in the scalae depend on the single spatial coordinate, $x$. Since the cochlea is surrounded by rigid bone and no net fluid flows into or out of the system at acoustic frequencies, the fluid volume velocities in the two scalae are constrained to be equal in magnitude but opposite in direction at all locations $x$. Although Eq. (A1) bears a strong resemblance to Webster's horn equation (e.g., Eisner 1967; Pierce 1981; Blackstock 2000), it differs from several previous treatments of the tapered cochlea in the literature (e.g., Fletcher 1953; de Boer 1980; Koshigoe et al. 1983). These treat- ments, however, violate energy conservation by implying that scalae tapering induces fluid flow in the absence of an applied pressure gradient.

We supplement Eq. (A1) with standard boundary conditions at the helicotrema $(x=L)$ and oval window $(x=0)$. At the helicotrema we assume that the pressure difference across the partition is zero $[p(L$, $t)=0]$; at the stapes we apply conservation of volume velocity in the scala vestibuli to obtain the condition

$$
\left.\frac{\partial p}{\partial x}\right|_{x=0}=-\rho_{0} S_{\text {ow }} \dot{v}_{\text {ow }} / S_{0}
$$

, where $S_{0}=S(0)$ and $S_{\mathrm{ow}}$ and $v_{\mathrm{ow}}(t)$ are, respectively, the oval-window area and velocity (positive into the scala vestibuli).

Equation (A1) can be simplified by introducing the change of variables $x \rightarrow \chi$ defined by

$$
\chi(x)=S_{0} \int_{0}^{x} \frac{d x^{\prime}}{S\left(x^{\prime}\right)}
$$

, where $\chi$ varies between 0 and $L_{\chi} \equiv \chi(L)$. The spatial variable $\chi$ represents the effective "acoustic distance", from the stapes; it increases more rapidly with $x$ when the scalae areas are small than when they are large and reduces to the conventional distance $x$ when the scalae areas are constant. All dependent variables are now regarded as functions of $\chi$. Equation (A1) becomes

$$
\frac{\partial^{2} p}{\partial \chi^{2}}=\rho_{0} b S \dot{v}_{\mathrm{bm}} / S_{0}^{2}
$$

, with boundary conditions $p\left(L_{\chi}, t\right)=0$ and

$$
\left.\frac{\partial p}{\partial \chi}\right|_{\chi=0}=-\rho_{0} S_{\text {ow }} \dot{v}_{\text {ow }} / S_{0}
$$

\section{Wave-equation formulation}

By eliminating terms in Eq. (A1) proportional to the first spatial derivative of the pressure, the change of variables allows the equation to be put in the form of a standard wave equation (e.g., Zweig et al. 1976; Shera and Zweig 1991b). For example, if we assume harmonic time dependence and write $p(\chi, t)=\mathrm{Re}$ $\left\{P(\chi, \omega) e^{i \omega t}\right\}$ and $v_{\mathrm{bm}}(\chi, t)=\operatorname{Re}\left\{V_{\mathrm{bm}}(\chi, \omega) e^{i \omega t}\right\}$, then Eq. (A5) assumes the form

$$
P^{\prime \prime}(\chi, \omega)+k_{\chi}^{2}(\chi, \omega) P(\chi, \omega)=0
$$

, where the prime denotes a derivative with respect to $\chi$ [cf. Eq. (18) of Talmadge et al. 1998]. The "wavenumber" (in $\chi$ space) is defined by

$$
k_{\chi}^{2}(\chi, \omega)=-Z(\chi, \omega) Y(\chi, \omega)
$$


where equation $Z(\chi, \omega)=\mathrm{i} \omega \rho_{0} S / S_{0}{ }^{2}$ is the longitudinal impedance of the fluids and $Y(\chi, \omega)$ is the admittance of the organ of Corti, defined so that $V_{\mathrm{bm}}=Y P / b$.

The general solution has the form [cf. Eq. (1)]

$$
P(\chi, \omega)=a_{+}(\omega) W_{+}(\chi, \omega)+a_{-}(\omega) \mathrm{W}_{-}(\chi, \omega)
$$

where the wave amplitudes $a_{ \pm}$are determined by boundary conditions and the pressure basis waves $W_{ \pm}(\chi, \omega)$ can be obtained by solving Eq. (A7) numerically or semianalytically using the WKB approximation (e.g., Shera and Zweig 1991a; Talmadge et al. 1998).

\section{Green's function formulation}

The hydrodynamic Green's function, $G(\chi, \bar{\chi})$, is defined as the solution to Eq. (A5) when the quantity on the right-hand side of the equality is a point source at location $\bar{\chi}$. In other words, $G(\chi, \bar{\chi})$ satisfies the equation

$$
\frac{\partial^{2} G(\chi, \bar{\chi})}{\partial \chi^{2}}=\frac{\partial^{2} G(\chi, \bar{\chi})}{\partial \bar{\chi}^{2}}=-\delta(\chi-\bar{\chi})
$$

subject to the boundary conditions $G\left(\chi, L_{\chi}\right)=0$ at the helicotrema and at $\bar{\chi}=0$. The function satisfying these constraints is easily seen to be

$$
G(\chi, \bar{\chi})=L_{\chi}-\max (\chi, \bar{\chi})
$$

where $\max (\chi, \bar{\chi})$ is the greater of its two arguments. $G(\chi, \bar{\chi})$ thus consists of two straight-line segments intersecting at $\chi=\bar{\chi}$ with a unit discontinuity in their slopes. Equations (A5) and (A6) for the pressure can now be written as an integral equation using the Green's function (e.g., Friedman 1956):

$$
\begin{aligned}
p(\chi, t) & =\frac{\rho_{0}}{S_{0}^{2}}\left[G(\chi, 0) S_{0} S_{\mathrm{ow}} \dot{v}_{\mathrm{ow}}(t)\right. \\
& \left.-\int_{0}^{L_{\chi}} b(\bar{\chi}) S(\bar{\chi}) G(\chi, \bar{\chi}) \dot{v}_{\mathrm{bm}}(\bar{\chi}, t) d \bar{\chi}\right]
\end{aligned}
$$

When rewritten using the conventional spatial variable $x$, Eq. (A12) becomes

$$
\begin{aligned}
p(x, t) & =\frac{\rho_{0}}{S_{0}}\left[G(x, 0) S_{\text {ow }} \dot{v}_{\text {ow }}(t)\right. \\
& \left.-\int_{0}^{L b(\bar{x}) G(x, \bar{x}) \dot{b}_{\mathrm{bm}}(\bar{x}, t) d \bar{x}}\right]
\end{aligned}
$$

where

$$
G(x, \bar{x}) \equiv G[\chi(x), \bar{\chi}(\bar{x})]=S_{0} \int_{\max (x, \bar{x})}^{L} \frac{d x^{\prime}}{S\left(x^{\prime}\right)}
$$

Equation (A13) is the one-dimensional version of Nobili et al.'s "BM integrodifferential equation" [cf.

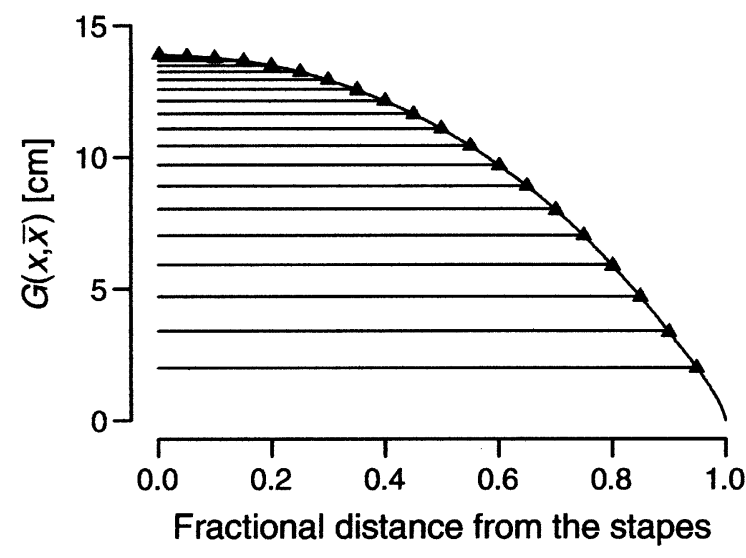

FIG. A1. One-dimensional hydrodynamic Green's functions for the guinea pig cochlea. The figure shows plots of $G\left(x, \bar{x}_{i}\right)$ at the 20 locations $\bar{x}_{i}$ identified by the black triangles $(\boldsymbol{\Delta})$. The Green's functions were computed from Eq. (A14) using anatomical measurements of scalae areas from Fernández (1952).

Nobili et al. 2003a, Eq. (1)]. The solution at angular frequency $\omega$ is equivalent to the wave-equation formulation [Eq. (A9)] evaluated with appropriate choice of wave amplitudes $a_{ \pm}$.

Green's functions for the guinea pig cochlea. Figure A1 shows plots of the Green's function $G(x, \bar{x})$ at various locations $\bar{x}$ computed for the guinea pig cochlea using anatomical measurements of scalae areas (Fernández 1952). In the box model, $G(x, \bar{x})$ is a straight, downward-sloping line for $x>\bar{x}$; the curvature evident in the figure arises as the result of scalae tapering. [In the tapered cochlea, it is $G(\chi, \bar{\chi})$, not $G(x, \bar{x})$, that consists of intersecting straight lines.] Except for small values of $|x-\bar{x}|$, the Green's function $G(x, \bar{x})$ for the one-dimensional box model [obtained by setting $S(x)=S_{0}$ in Eq. (A14)] has essentially the same form as the Green's function derived by Allen (1977) for the two-dimensional box model (cf. Allen's Fig. 4).

In their work, Nobili et al. (e.g., Mammano and Nobili 1993; Nobili et al. 2003a) adopt a slightly different definition of the Green's function based on the hydrodynamic force pushing on the BM rather than on the pressure difference across its surface [cf. Eq. (1) of Nobili et al. 2003a]. The differing definitions imply that

$$
G_{\mathrm{N}}(x, \bar{x})=\rho_{0} b(x) b(\bar{x}) G(x, \bar{x}) / S_{0}
$$

where $G_{\mathrm{N}}(x, \bar{x})$ is the long-range component of Nobili et al.'s Green's function (or "force propagator"). Figure A2 shows plots of $G_{\mathrm{N}}(x, \bar{x})$ computed for the guinea pig from Eq. (A15) using Fernández's measurements of BM width. Except for the absence of a "spike" at $x=\bar{x}$ arising from higher-dimensional effects (and a minor but unexplained difference in overall normalization), the results bear a strong 


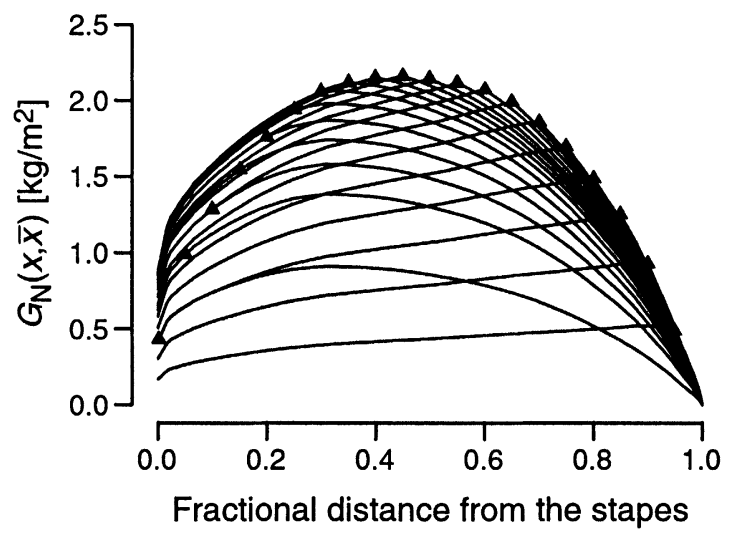

FIG. A2. One-dimensional long-range force propagators for the guinea pig cochlea. The figure shows plots of $G_{N}\left(x, \bar{x}_{i}\right)$ at the 20 locations $\bar{x}_{i}$ identified by the black triangles $(\boldsymbol{\Lambda})$. Force propagators were computed from Eq. (A15) by combining the results shown in Figure A1 with anatomical measurements of BM width from Fernández (1952). Apart from an unaccounted-for difference in normalization, $G_{N}(x, \bar{x})$ is functionally identical to the long-range Green's function computed by Mammano and Nobili (1993) for their three-dimensional model (cf. their Fig. A3). Small discrepancies between the plots presumably result from differences in digitizing and interpolating Fernández's anatomical measurements.

resemblance to Figure A3 of Mammano and Nobili (1993). Indeed, close inspection of their formulae [i.e., their Eq. (A5) with the substitutions given in the succeeding paragraphs] indicates that the two are functionally identical.

In this Appendix we have illustrated the connection between cochlear geometry and the nature of the long-range part of the Green's function $G(x, \bar{x})$ appearing in Eq. (A13). The fact that the long-range fluid coupling described by $G(x, \bar{x})$ varies nonlinearly with $x$ (and thus "cannot be canceled by multiple differentiation") does not imply the absence of an underlying wave equation, as asserted by Nobili et al. (e.g., Mammano and Nobili 1993). After the BM acceleration is related to $p(x, t)$ via cochlear dynamics, the underlying wave equation in the tapered cochlea [i.e., Eq. (A1) or its equivalent, Eq. (A5)] is a variant of Webster's horn equation, rather than the simpler wave equation that results in the box model, where $S(x)$ is constant.

\section{REFERENCES}

Allen JB. Two-dimensional cochlear fluid model: New results. J. Acoust. Soc. Am. 61:110-119, 1997.

Allen JB. Moderated discussion of cochlear biophysics. In: GuMmer AW (eds) Biophysics of the Cochlea: From Molecules to Models. World Scientific, Singapore, pp 563-592, 2003.

BÉKÉsY G. von. Experiments in Hearing. McGraw-Hill, New York, 1960.

Blackstock DT. Fundamentals of Physical Acoustics. John Wiley \& Sons, New York, 2000.
Dallos P. The Auditory Periphery: Biophysics and Physiology. Academic Press, New York, 1973.

Boer E. DE. Auditory physics. Physical principles in hearing theory. I. Phys. Rep. 62:88-174, 1980.

Boer E. DE. Auditory physics. Physical principles in hearing theory. II. Phys. Rep. 105:142-226, 1984.

EISNER E. Complete solutions of the 'Webster' horn equation. J. Acoust. Soc. Am 41:1126-1146, 1967.

Fernández C. Dimensions of the cochlea (guinea pig). J. Acoust. Soc. Am. 24:519-523, 1952.

Fletcher H. Speech and Hearing in Communication, ASA Edition (1995) Acoustical Society of America, Woodbury, NY, 1953.

Friedman B. Principles and Techniques of Applied Mathematics. Wiley, New York, 1956.

KEMP DT. The evoked cochlear mechanical response and the auditory microstructure-Evidence for a new element in cochlear mechanics. Scand. Audiol. Suppl. 9:35-47, 1979.

Koshigoe S, Kwok WK, Tubis A. Effects of perilymph viscosity on lowfrequency intracochlear pressures and the cochlear input impedance of the cat. J. Acoust. Soc. Am. 74:486-492, 1983.

Lighthill J. Energy flow in the cochlea. J. Fluid Mech. 106:149-213, 1981.

Mammano F, Nobili R. Biophysics of the cochlea: Linear approximation. J. Acoust. Soc. Am. 93:3320-3332, 1993.

Nobili R. Otoacoustic emissions simulated by a realistic cochlear model. In: WADA H, TAKASAKa T, IKeda K, OHYAMA K, KoIKe T (eds) Recent Developments in Auditory Mechanics. World Scientific, Singapore, pp 402-408, 2000.

Nobili R, Mammano F, Ashmore J. How well do we understand the cochlea? Trends Neurosci 21:159-167, 1998.

Nobili R, Veteáník A, Turicchia L, Mammano F. Otoacousstic emissions from residual oscillations of the cochlear basilar membrane in a human ear model. J. Assoc. Res. Otolaryngol. 4:478494, 2003a.

Nobili R, Veteáník A, Turicchia L, Mammano F. Otoacoustics emissions simulated in the time domain by a hydrodynamic model of the human cochlea. In: Gummer AW (ed) Biophysics of the Cochlea: From Molecules to Models. World Scientific, Singapore, pp 524-530, 2003b.

Peterson LC, Bogert BP. A dynamical theory of the cochlea. J. Acoust. Soc. Am. 22:369-381, 1950.

Pierce AD. Acoustics: An Introduction to its Physical Principles and Applications. McGraw-Hill, New York, 1981.

REN T. Longitudinal pattern of basilar membrane vibration in the sensitive cochlea. Proc. Natl. Acad. Sci. USA. 99:17101-17106, 2002.

SHERA CA. Mammalian spontaneous otoacoustic emissions are amplitude stabilized cochlear standing waves. J. Acoust. Soc. Am. 114:244-262, 2003.

Shera CA, Zweig G. Reflection of retrograde waves within the cochlea and at the stapes. J. Acoust. Soc. Am. 89:1290-1305, 1991a.

Shera CA, Zweig G. A symmetry suppresses the cochlear catastrophe. J. Acoust. Soc. Am. 89:1276-1289, 1991b.

Shera CA, Zweig G. Order from chaos: Resolving the paradox of periodicity in evoked otoacoustic emission. In: Duifhuis $H$, Horst JW, van Dijk P, van Netten SM (eds) Biophysics of Hair Cell Sensory Systems. World Scientific, Singapore, pp 54-63, 1993.

Shera CA, Tubis A, Talmadge CL. Coherent reflection in a $2+1$ dimensional cohlea: Short-wave versus long-wave scattering in the generation of reflection-source otoacoustic emissions (in preparation) 2004.

Talmadge CL, Tubis A. On modeling the connection between spontaneous and evoked otoacoustic emissions. In: DuifHuIs H, Horst JW, van Dijk P, van Netten SM (eds) Biophysics of Hair Cell Sensory Systems. World Scientific, Singapore, pp 25-32, 1993. 
Talmadge CL, Tubis A, Tong C. Cochlear wave reflection due to roughness in 2-d and 3-d cochlear models. Assoc. Res. Otolaryngol. Abst. 24:44.

Talmadge CL, Tubis A, Long GR, Piskorski P. Modeling otoacoustic emission and hearing threshold fine structures. J. Acoust. Soc. Am. 104:1517-1543, 1998.

Talmadge CL, Tubis A, Long GR, Tong C. Modeling the combined effects of basilar membrane nonlinearity and roughness on stimulus frequency otoacoustic emission fine structure. J. Acoust. Soc. Am. 108:2911-2932, 2000.

Wegel RL, LANE CE. The auditory masking of one pure tone by another and its probable relation to the dynamics of the inner ear. Phys. Rev. 23:266-285, 1924.
Wнiтнам GB. Linear and Nonlinear Waves. John Wiley \& Sons, New York, 1974.

ZweIG G. Finding the impedance of the organ of Corti. J. Acoust. Soc. Am. 89:1229-1254, 1991.

ZwEIG G, SHERA CA. The origin of periodicity in the spectrum of evoked otoacoustic emissions. J. Acoust. Soc. Am. 98:2018-2047, 1995.

Zweig G, Lipes R, Pierce JR. The cochlear compromise. J. Acoust. Soc. Am. 59:975-982, 1976.

ZWISLOCKI J. Analysis of some auditory characteristics. In: LuCE RD, Bush RR, Galanter E (eds) Handbook of Mathematical Psychology. John Wiley \& Sons, New York, pp 1-97, 1965.

Zwislocki-Mościcki J. Theorie der Schneckenmechanik: Qualitative und quantitative Analyse. Acta Otolaryngol. Suppl. 72:1-112, 1948. 